\title{
Analysis of 140 Consecutive Autopsy Cases of Cerebrovascular Strokes in Northern Japan*
}

\author{
Hitoshi Fukasawa \\ Department of Pathology, Research Institute of Brain and Blood \\ Vessels, Akita.
}

\begin{abstract}
Fukasawa, H. Analysis of 140 Consecutive Autopsy Cases of Cerebrovascular Strokes in Northern Japan. Tohoku J. exp. Med., 1975, 117 (4), 357-372On the autopsy findings of the 140 consecutive stroke cases, some characteristics of cerebrovascular diseases in this district were discussed. Predominance of intracranial hemorrhage (cerebral hemorrhage and subarachnoid hemorrhage) over cerebral infaretion was still evident. Ruptured intracranial aneurysms were demonstrated in $98 \%$ of fatal subarachnoid hemorrhage. Compared with the autopsy data of other institutions, the age of death was generally low, the median age for cerebral hemorrhage, subarachnoid hemorrhage and cerebral infarction falling in the fourth, fifth, and sixth decade, respectively. Cerebellar hemorrhage was relatively frequent, while blood dyscrasias and other symptomatic hemorrhage constituted only a small part in contrast with the report of American authors. A dominant role of hypertension in causing strokes was concluded from both the heart weight and the clinical records. - cerebral hemorrhage; intracranial aneurysm; cerebral infarction; stroke; hyportension
\end{abstract}

It is well-known that cerebrovascular strokes are the major cause of death in the Japanese population; the predominant role of the diseases is particularly remarkable in the northern part of the main island, especially in Akita, where our Research Institute is located. Although the studies conducted by Katsuki and his colleagues in Hisayama village (Katsuki and Hirota 1966) made a valuable contribution to the epidemiological study of strokes in the Western Japan, it is not certain whether the result can be applied to the whole population of Japan or not, because the western and the northern part of the country are much different from each other in environments and in mode of life. The pathology of cerebrovascular diseases in this district, besides clinical and epidemiological investigation of the diseases, has interested us since the establishment of the Institute in 1969. In the present study, statistical analysis of the pathology is presented in 140 consecutive autopsy cases of strokes in Akita district.

\section{Materials and Metrhods}

The number of autopsy cases and inhospital autopsy percentage during the past five years are summarized in Table 1. Of these, 204 consecutive cases before June 1973

Received for publication, July 22, 1975.

* Presented at the 14th Local Assembly of the Japanese Society of Neurology, Sendai, February 21, 1974. 
TABLE 1. Number of autopsy cases

\begin{tabular}{lcccccc}
\hline & 1969 & 1970 & 1971 & 1972 & 1973 & Total \\
\hline Inhospital death & 26 & 50 & 51 & 52 & 65 & 224 \\
Inhospital autopsy & 21 & 45 & 48 & 48 & 54 & 216 \\
Autopsy percentage & $81 \%$ & $90 \%$ & $94 \%$ & $92 \%$ & $83 \%$ & $89 \%$ \\
Out-hospital autopsy & 1 & 6 & 9 & 7 & 2 & 25 \\
\hline \multicolumn{1}{c}{ Autopsy total } & 22 & 51 & 57 & 55 & 56 & 241 \\
\hline
\end{tabular}

(May, 1969 - December, 1973)

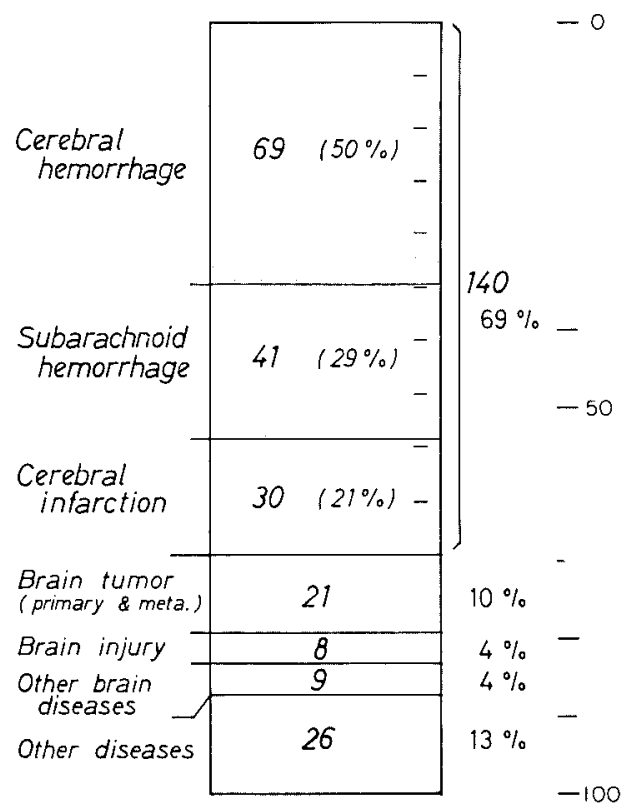

Fig. 1. Classification of autopsy cases according to main anatomical diagnosis (May, 1969 June, 1973). As the cause of death, only the major disease is taken into consideration for each individual. For example, if there were cerebral hemorrhage and old infarction, the case is included under the item of cerebral hemorrhage. Here "cerebral" is used synonymously with "eneephalic". $\square$ associated with old cerebral infaret.

were used for histological examination; the classification according to the main anatomical diagnosis is given in Fig. 1. Cerebrovascular strokes occupied as much as $70 \%$ of the total autopsy cases. The present study is based on 140 consecutive stroke cases in the autopsy series mentioned above.

The brains were fixed in $20 \%$ formalin for a period of two to three weeks, dissected in about $1 \mathrm{~cm}$ thick slices and examined by macroscopical observation. Histological sections were stained with hematoxylin and eosin, Masson's trichrome, luxol fast blue and cresyl-violet for myelin sheaths, and Bodian's silver impregnation for axons. Cajal's gold sublimate method for astrocytes and Holzer's methods for glial fibers were also applied to some cases. In selected cases, the brains were submitted to post-mortem angiographic examinations. 
RESULT

Age distribution of 140 autopsy cases of strokes is given in Fig. 2. Cerebral hemorrhages, most of which were of hypertensive origin, occurred predominantly in the later decades of life with an incidence peak around the ages of 50 to 60 . More than $80 \%$ of all deaths from cerebral hemorrhage fell between ages 40 and 70 . Subarachnoid hemorrhage (SAH) in this series was all due to ruptured intracranial aneurysm except in one case. Compared with the age distribution of cerebral hemorrhage, the curve is shifted to the lower ages by about 10 years and the age peak was somewhat blurred. Incidence of fatal cerebral infarction had a tendency toward increase with advancing age. The median age of death for cerebral hemorrhage was 54, for $\mathrm{SAH} 49$, and for cerebral infarction 67 . The ratio of fatal intracerebral hemorrhage to infarction under 70 years of age was $3.8: 1$.

\section{Cerebral hemorrhage}

In this study, cerebral hemorrhage was diagnosed in cases of clinical symptoms of stroke when the autopsy revealed hematomas at least $2 \mathrm{~cm}$ in their largest dimen-

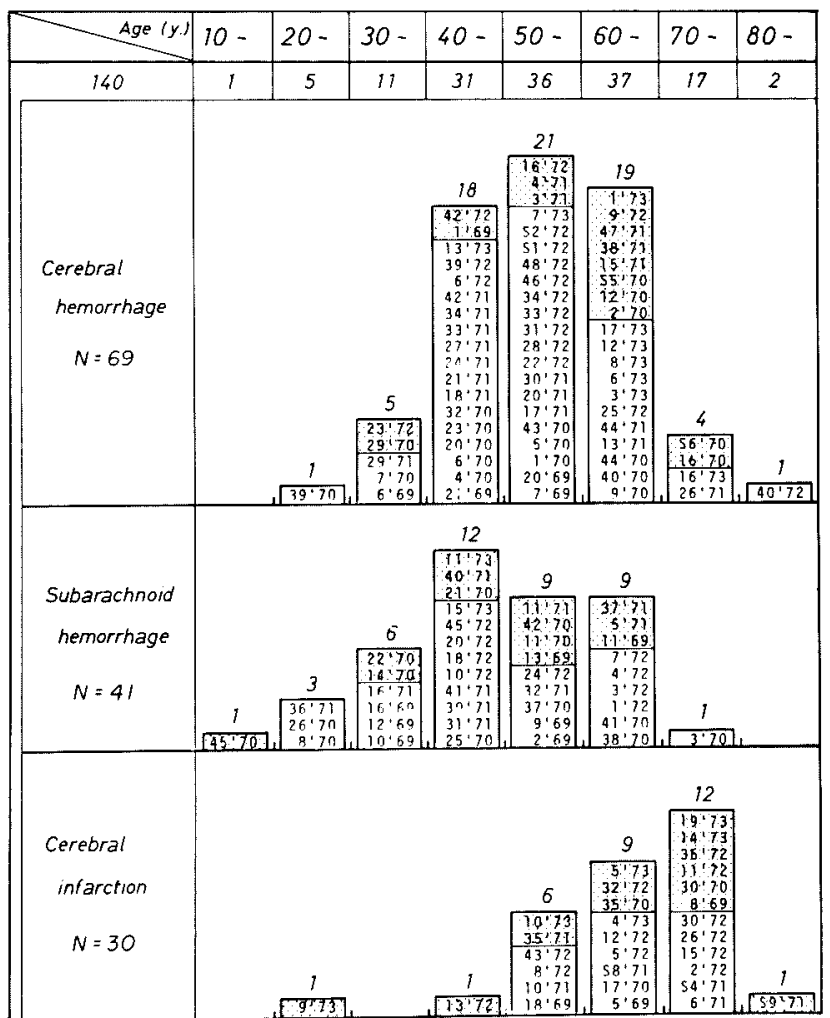

Fig. 2. Age distribution of consecutive autopsy cases of strokes (May, 1969 June, 1973). The ratio of fatal intracerebral hemorrhage to infarction is $2.3: 1$. $\square$ male $(N=96)$; $\square$ female $(N=44)$. The digits in the column refer to the autopsy number of the Institute. 


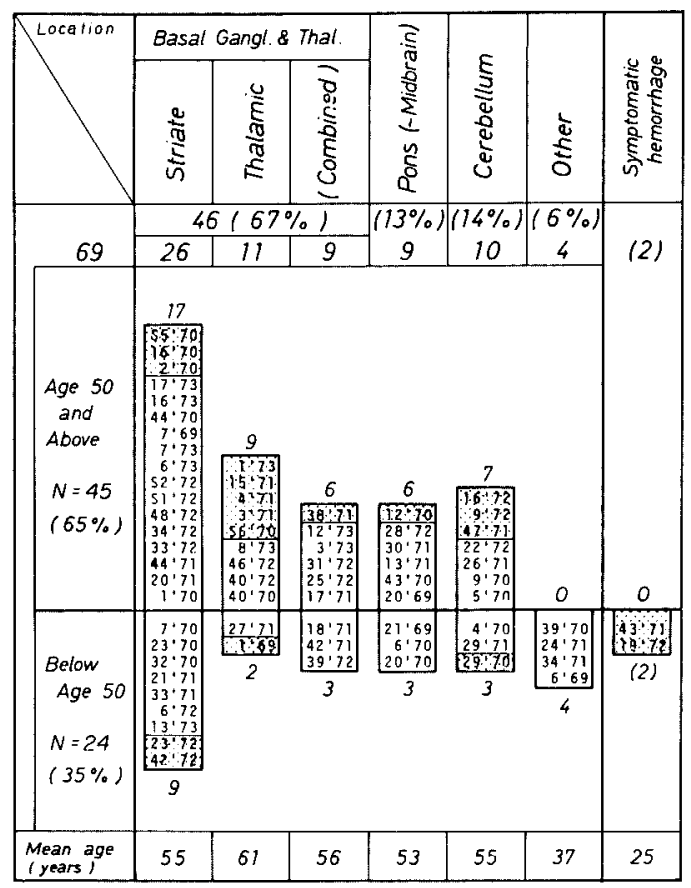

Fig. 3. Location of cerebral hemorrhage. Two cases listed under "symptomatic" hemorrhage are those of leukemia and toxemia of pregnancy. $\square$ male $(N=52)$; $\square$ female $(N=17)$.

sion in the cerebral hemisphere and $1 \mathrm{~cm}$ in the cerebellum or brain stem. Cases of cerebral hemorrhage secondary to neoplasms or blood dyscrasias were not included. The location of the cerebral hemorrhage was classified in six groups: (1) striate, (2) thalamic, (3) "combined" (or advanced), (4) brain stem (pons or midbrain), (5) cerebellar and (6) other sites. The results are given in Fig. 3. Thirty-five percent of the cases in our series of fatal hemorrhage were those under 50 years of age. Cerebellar hemorrhage made a higher percentage (14\%) than usually reported (Stehbens 1972). The mean age of death from thalamic hemorrhage in this series was some 8 years higher than that from pontine hemorrhage (53 years of age); the difference, however, was not statistically significant. Cerebral hemorrhage in "other sites" included, for instance, primary hematoma in the cerebral white matter, and such unusual locations were characteristic of cerebral hemorrhage in young individuals under the age of 50 .

Our histological findings of the cerebral hemorrhage were generally in agreement with those of Ooneda (1974); in some instances, pathogenesis of hemorrhage was attributable to the rupture of the intracerebral microaneurysms (Fig. 4) resulting from fibrinoid necrosis or plasmatic arterionecrosis as reported by Ooneda and his associates (1973). However, the percentage of their occurrence 


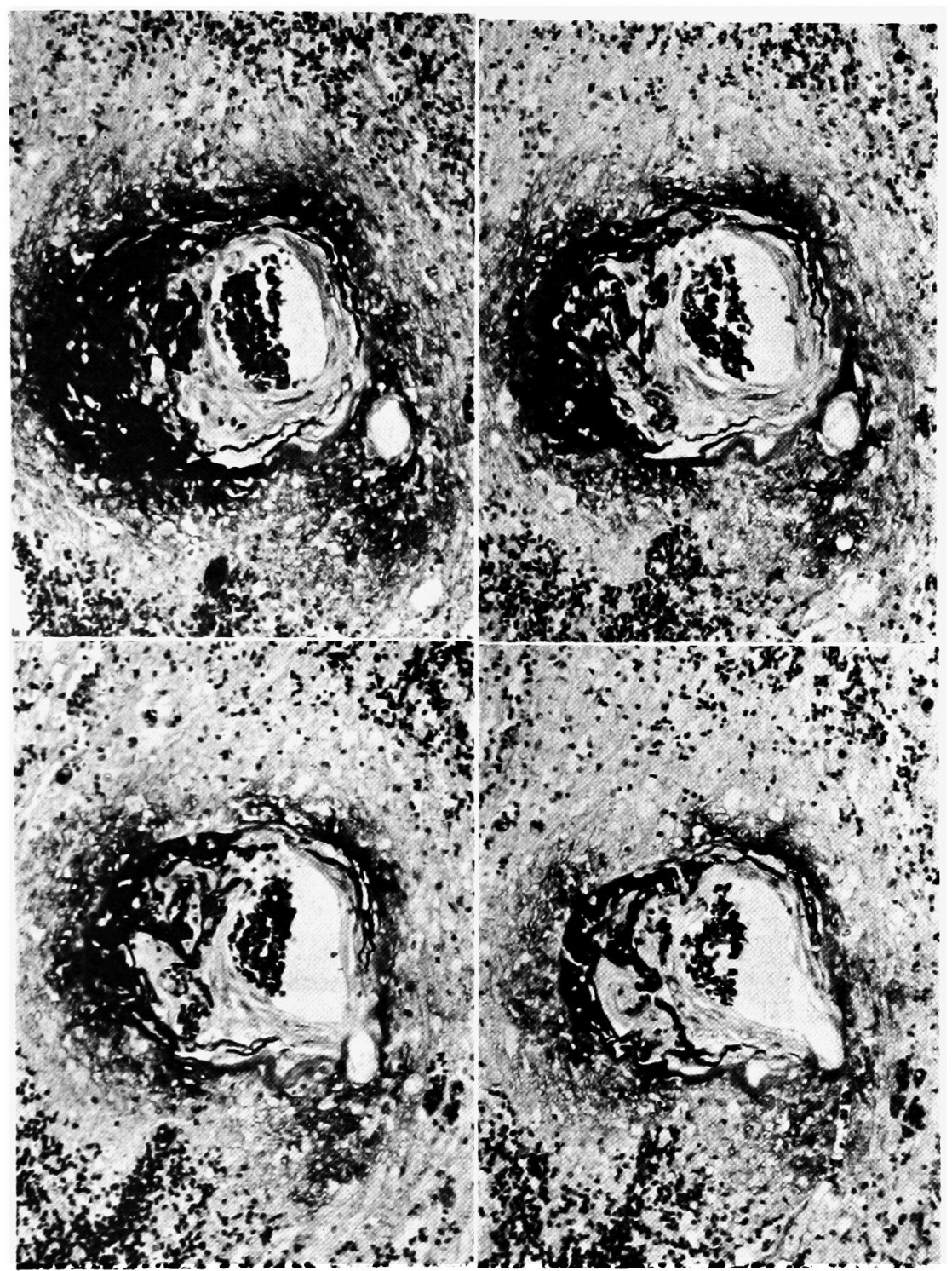

Fig. 4. Arterionecrotic microaneurysm with the wall exhibiting considerable fibrin infiltration, demonstrated by serial sections in a focus of pontine hemorrhage.

was not estimated, because only a limited number of cases in this series were examined by the tissue clearing method for microaneurysms.

\section{Subarachnoid hemorrhage (Ruptured intracranial aneurysms)}

In 40 of 41 cases of fatal subarachnoid hemorrhage ruptured intracranial saccular aneurysms were demonstrated. In the other one, bleeding sources could 


\begin{tabular}{|c|c|c|}
\hline$I$ & II & III \\
\hline $\begin{array}{l}\text { Bleeding into } \\
\text { the Subarach- } \\
\text { noid Space } \\
\text { (Retrograde Extension } \\
\text { of Blood into the } \\
\text { Ventricular System) }\end{array}$ & $\begin{array}{l}\text { Disruption of the Cerebral } \\
\text { Tissue } \\
\text { Bleeding into the Ventricular } \\
\text { System }\end{array}$ & $\begin{array}{l}\text { Formation of a Hematoma } \\
\text { in the Cerebral Sulcus } \\
\text { Necrosis of the Adjacent } \\
\text { Cerebral Tissue } \\
\text { Secondary Intraventricular } \\
\text { Hemorrhage }\end{array}$ \\
\hline $\begin{array}{l}\text { Ventricular enlarge. } \\
\text { ment }(-) \sim(+) \text { (bilat.) }\end{array}$ & $\begin{array}{l}\text { Ventricular enlargement:(-) }-(+) \\
\text { (unilat.) }\end{array}$ & $\begin{array}{l}\text { Ventricular compression } \\
\text { displacement }\end{array}$ \\
\hline Massive $S A H$ & $\underset{\substack{\text { Ant horn, } \\
\text { II ventricle }}}{a}$ & suic. of cor.cal. \\
\hline Minimal SAH & $\stackrel{4}{ \pm} I^{\prime}$ & \\
\hline
\end{tabular}

Fig. 5. Types of blood invasion into the brain from ruptured aneurysms. Originally presented by Hirano (1961) and modified by the author.

not be identified. The patterns of intracranial hemorrhage following ruptured aneurysms were classified into 3 major types (Figs. 5 and 6):

I) Pure subarachnoid hemorrhage,

II) Intracerebral and subarachnoid hemorrhage, and

III) Subarachnoid hematoma with cerebral necrosis.

The relation between the location of the aneurysms and the blood extension pattern is shown in Fig. 7. Death within a week after the onset was more frequent in Types II and III. About $45 \%$ of the aneurysms were located in the vicinity of the anterior communicating artery. As reported by many authors, a high incidence of saccular aneurysms in the anterior half of the circle of Willis was confirmed again in our series. The size of the aneurysm itself was not correlated with the grade of hemorrhage.

\section{Cerebral infarction}

In our series of 30 cases of cerebral infarction, occlusion of the major brain arteries was demonstrated at autopsy in $60 \%(18 / 30)$. The site most frequently involved was the internal carotid (10 cases) and the next most frequent site was the middle cerebral artery (4 cases). The other cases were those without complete obstruction of large arteries. The size and shape of the infarction were not uniform, even if the site of vascular occlusion was apparently similar.

4. The time interval between onset of stroke and death

Fig. 8 shows the length of survival in 140 autopsied cases of strokes. For 


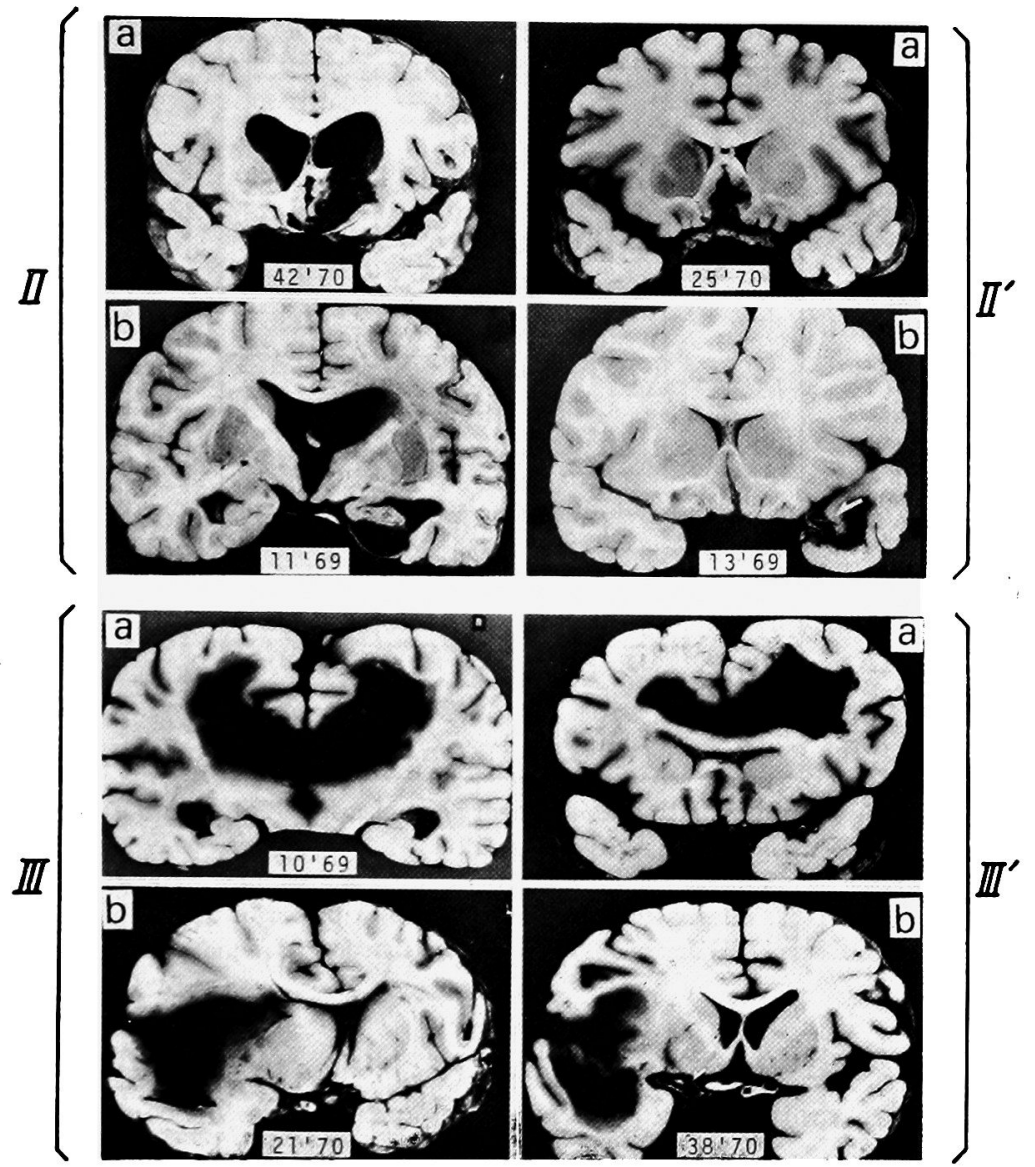

Fig. 6. Types of intracerebral blood invasion from ruptured intracranial aneurysms.

fatal cerebral hemorrhage, the mortality rate within the first week was $42 \%$, for SAH $39 \%$ and for cerebral infarction $23 \%$. Thus, of these three major cerebrovascular diseases the patients with cerebral hemorrhage died earlier than those with the other two diseases. In particular, there was a 10\% mortality in the first 24 $\mathrm{hr}$ for thalamic, pontine, and cerebellar hemorrhage. On the other hand, even massive pontine hemorrhage may be compatible with life over a month under an adequate control of respiration (Fig. 9).

\section{Associated lesions of cerebrovascular strokes}

Ventricular rupture (Fig. 10). More or less pronounced ventricular hemorrhage was demonstrated in $68 \%$ of the cases of cerebral hemorrhage, while $41 \%$ of SAH was associated with the pathological finding, and only $3 \%$ of cerebral infarction had ventricular hemorrhage. The difference between cerebral hemorrhage and the 


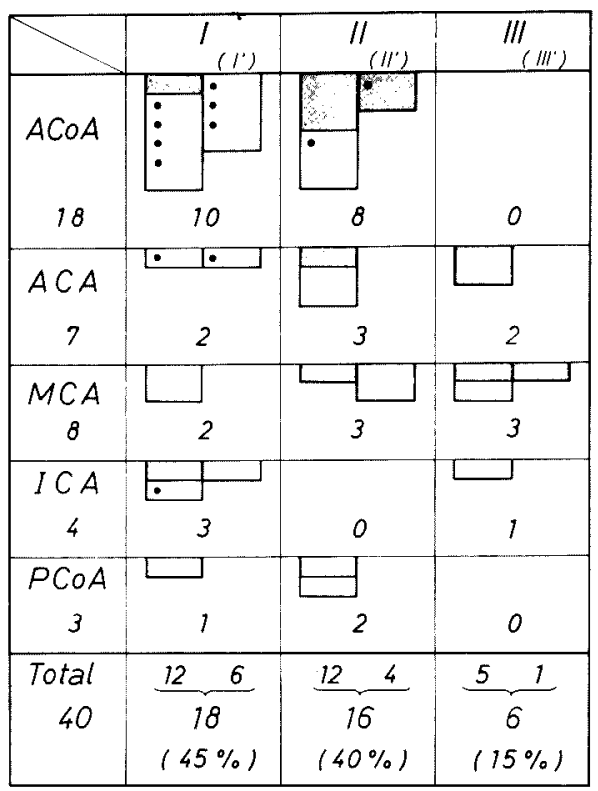

Fig. 7. Location of ruptured intracranial aneurysms classified into three types according to the extension of hemorrhage (See Fig. 5).

$\mathrm{ACoA}$, anterior communicating artery; ACA, anterior cerebral artery; MCA, middle cerebral artery; ICA, internal carotid artery; $\mathrm{PCoA}$, posterior communicating artery. survival $\leq 1$ week; • operated by clipping or ligation of aneurysm.

other two diseases is statistically significant $(p<0.05)$. In basal ganglionic hemorrhage, ventricular rupture could play an important role in causing death. In SAH, however, ventricular bleeding was of minor importance as a direct cause of death. In cerebral infarction, rupture into the ventricle was observed in only one case of hemorrhagic infarction due to embolism.

Secondary brain stem hemorrhage (Fig. 11). Secondary brain stem hemorrhage is regarded as one of the immediate causes of death in cerebrovascular strokes. The incidence of this condition is summarized in Fig. 11. This was observed in $27 \%$ of the fatal cerebral hemorrhage and in $12 \%$ of the deaths from SAH. However, this difference in incidence was not statistically significant. Furthermore, the incidence in cerebral infarction was not much different from that in SAH. In brain diseases other than stroke, secondary brain stem hemorrhage was also found in $9 \%$ of the cases. Accordingly, the occurrence of secondary hemorrhage in the brain stem seemed to be related less to the nature of the primary lesion than to its rapidity of expansion. Its exact pathogenesis is still to be examined.

Complication of cerebral infarction (Fig. 12). Rates of co-existence of infarction scars recognizable at autopsy in cases of fresh cerebral infarction and cerebral hemorrhage were both approximately $17 \%$. In SAH, however, cases associated 


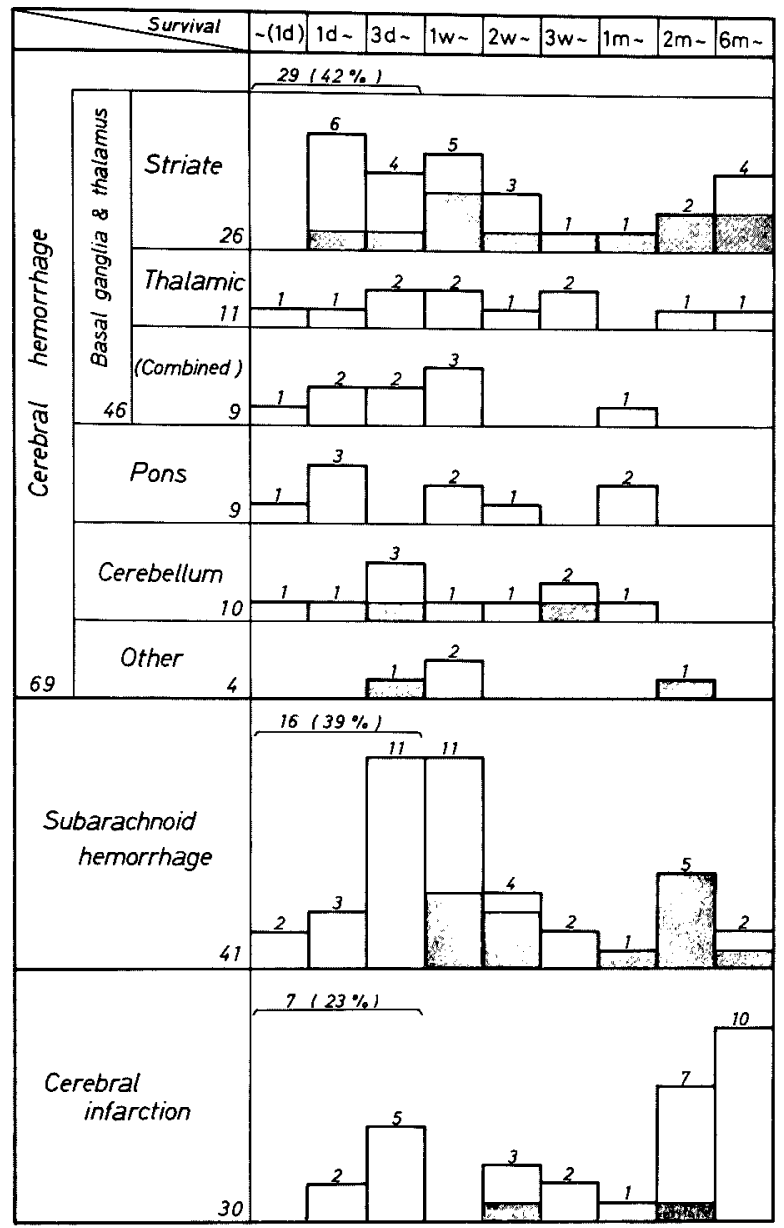

Fig. 8. Duration from the onset of stroke to death. The rate of death in the first week to that of total mortal cases of cerebral hemorrhage is $42 \%$, while those of SAH and cerebral infarction were $39 \%$ and $23 \%$. For the person with recurrent strokes, survival was calculated from the last attack. operated cases.

with such scars were only $5 \%$, a rate which is very close to that in diseases other than stroke.

The occurrences of fresh cerebral infarction secondary to cerebral hemorrhage and $\mathrm{SAH}$ were $9 \%$ and $34 \%$ respectively, the difference of which was statistically significant $(p<0.05)$. Even when the operated cases were excluded, the result was not greatly influenced.

Extracerebral complications. Foci of bronchopneumonia (focal consolidation greater than a centimeter in diameter) were detected at autopsy in approximately $50 \%$ of both the cases of cerebral hemorrhage and those of cerebral infarction (Fig. 13). This rate is nearly equal to that found in other brain diseases. Pulmo- 


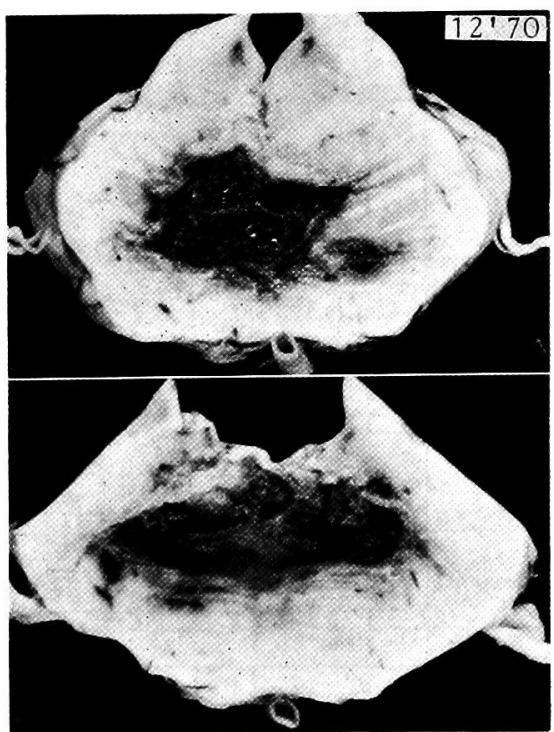

Fig. 9. An old hematoma of pons. Note capsule surrounding hematoma. 64-year-old female, 53 days' survival.

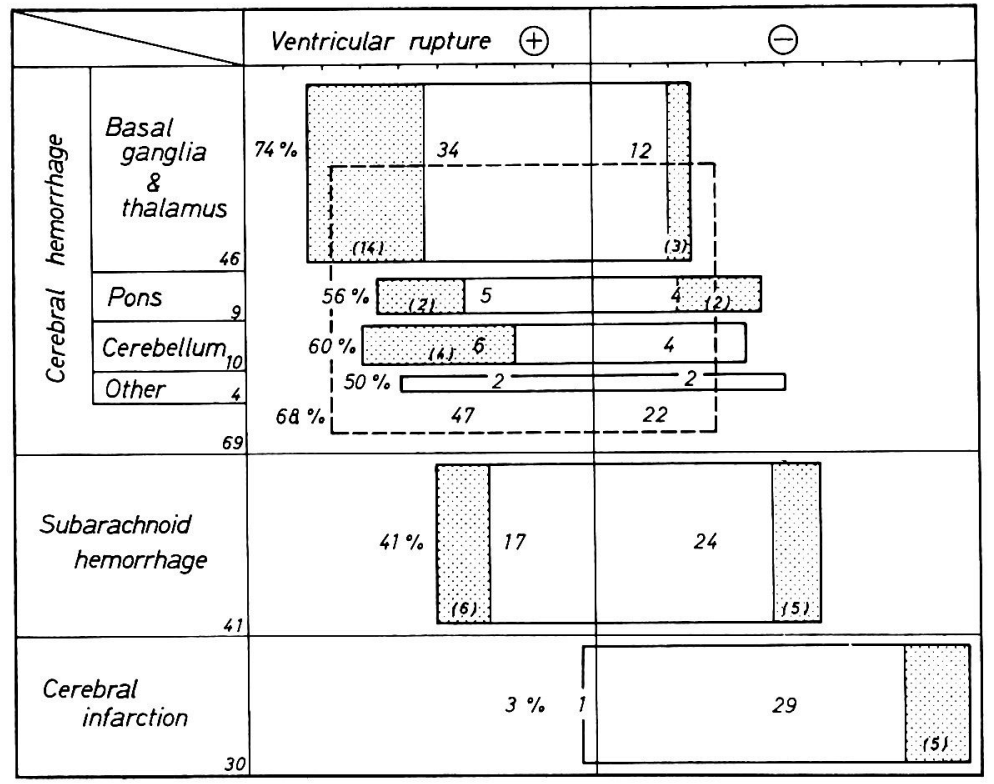

Fig. 10. Incidence of ventricular rupture. survival less than 5 days.

nary edema with foamy exudate and with lung weight over $900 \mathrm{~g}$ was demonstrated more frequently in $\mathrm{SAH}(24 \%)$ than in cerebral hemorrhage $(12 \%)$, though the difference is not statistically significant.

Hemorrhagic erosions or acute ulcers of the gastric mucosa were found in $39 \%$ 


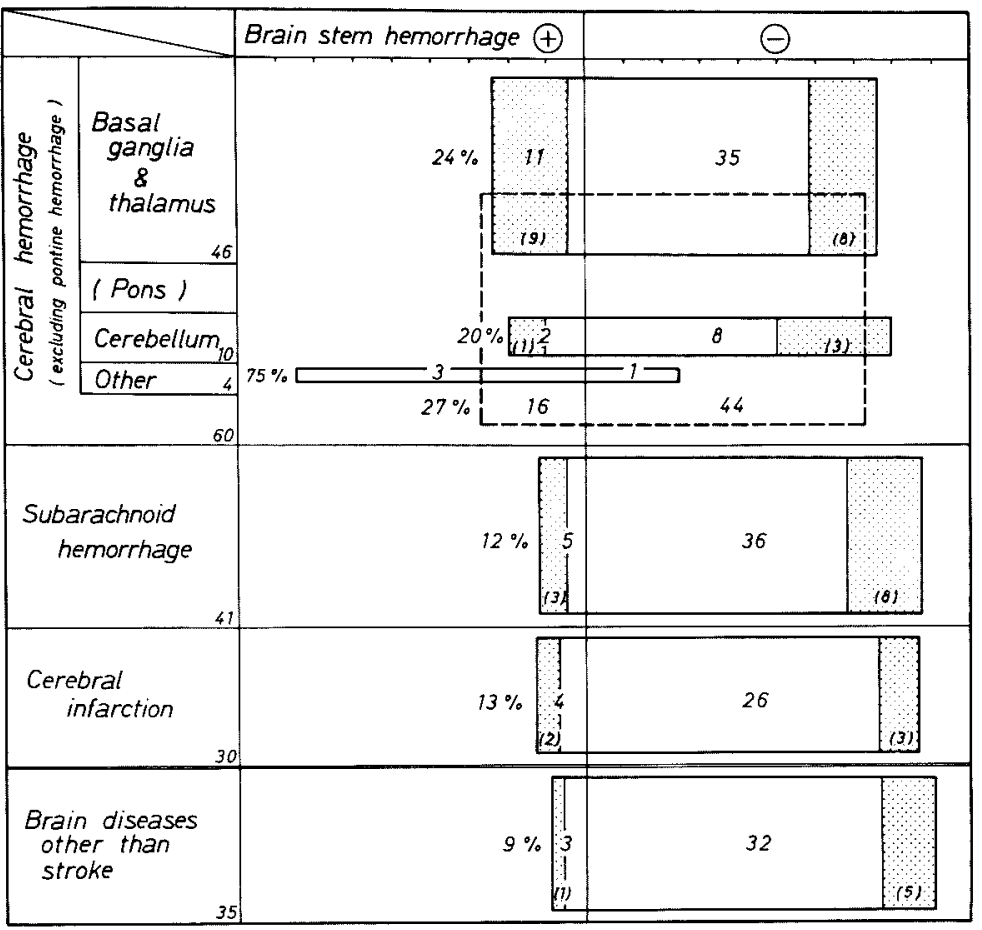

Fig. 11. Incidence of secondary brain stem hemorrhage.

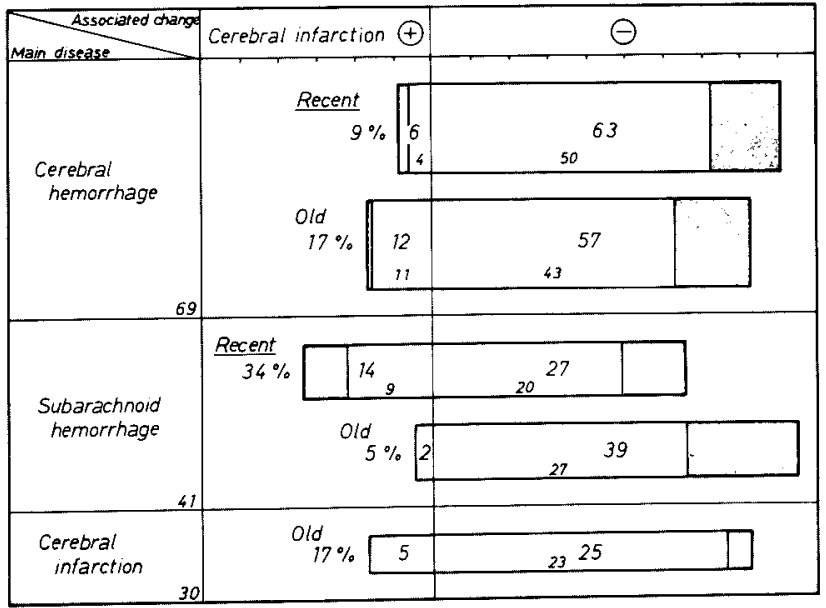

Fig. 12. Cerebral infarction as an associated change.

Recent: cerebral infarction secondary to cerebral or subarachnoid hemorrhage.

Old: cerebral infarction prior to the onset of the main disease.

operated cases.

Incidence of recent infarction in association with $\mathrm{SAH}$ is significantly higher than that with cerebral hemorrhage. 


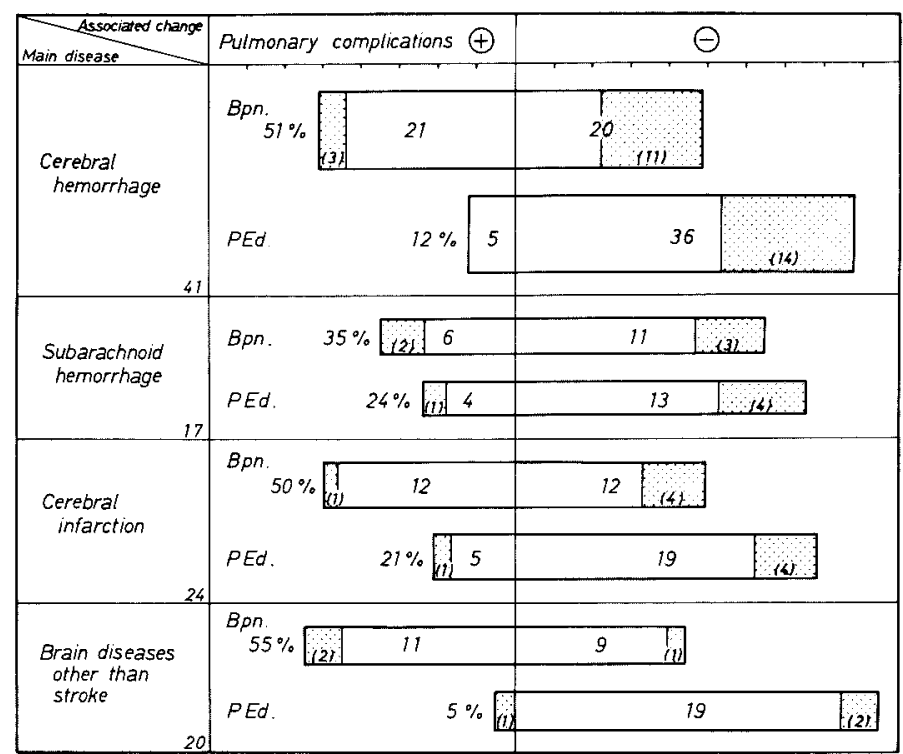

Fig. 13. Pulmonary complications. Bronchopneumonia was present in nearly $50 \%$ of both cerebral hemorrhage and cerebral infarction. Bpn., bronchopneumonia; PEd., pulmonary edema (lung weight $>900 \mathrm{~g}$ ). survival less than 1 week.

of the cases of cerebral hemorrhage (Fig. 14). The relation between severe gastic bleeding and the localization of intracerebral hematoma has not yet been established. It is confirmed, however, that the incidence rate of gastric hemorrhage in stroke patients is significantly higher than that in patients who died from non-cerebral diseases.

old myocardial infaretion and renal infarction were demonstrated in 29 and $21 \%$, respectively, of the 24 cases of cerebral infarction, while in 41 cases of cerebral

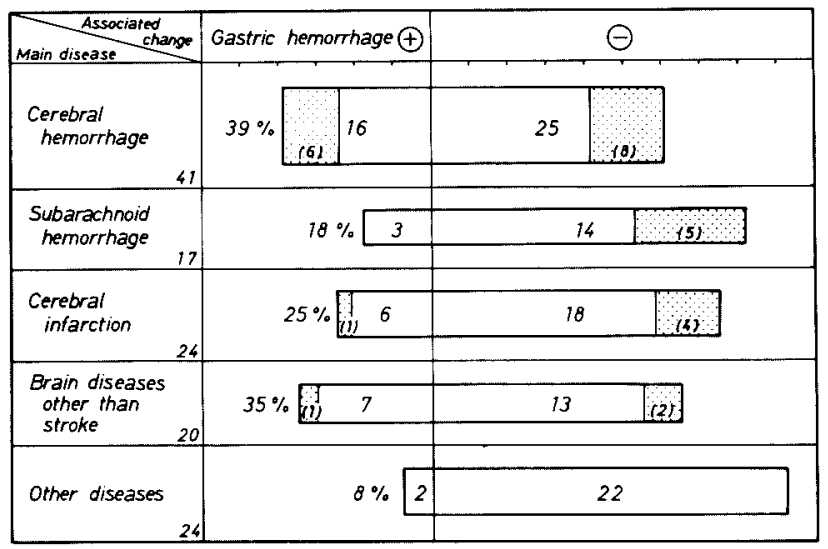

Fig. 14. Complication of hemorrhagic erosions (or ulcers) of the gastric mucosa. nurvival less than 1 week. 
hemorrhage there was no evidence of extracerebral infarction except in one case of small myocardial scar.

Distribution of heart weight by age in cerebral hemorrhage, cerebral infarction, $\mathrm{SAH}$, and other diseases in our autopsy series is illustrated in Fig. 15; and the mean heart weight over age 30 in each group is given in Table 2. Since cardiac valvular or primary myocardial diseases are not included, increased heart weight due to left ventricular hypertrophy is considered to indicate sustained systemic hypertension. It is clear from this result that hypertension plays an important role in causing fatal SAH as well as fatal cerebral hemorrhage.
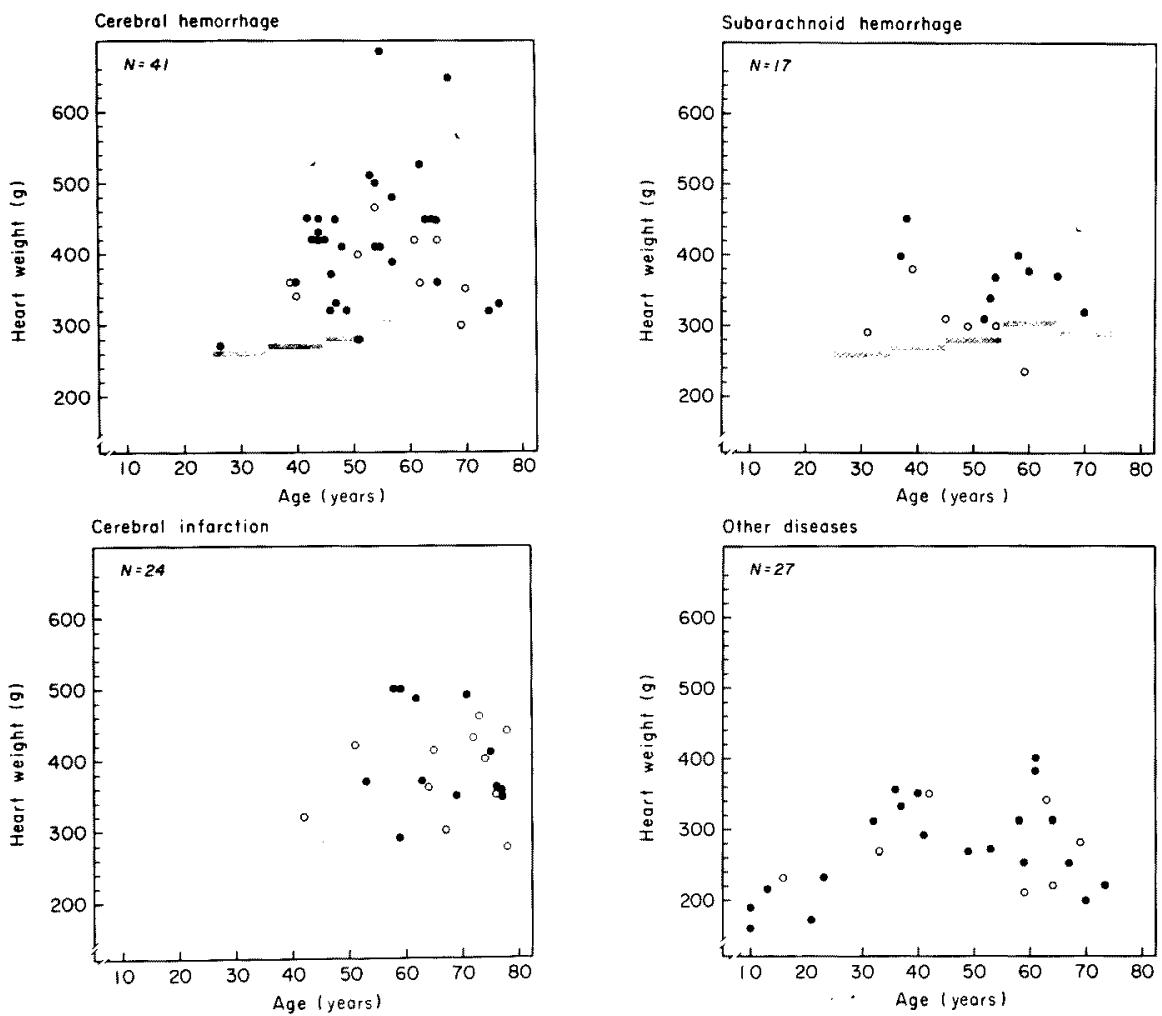

Fig. 15. Distribution of heart weights by age of autopsy cases of strokes and other diseases (consecutive general autopsy cases from May, 1969 to June, 1973). Bars in the figure represent the mean heart weight of Japanese male in each age group. 0 male; $\bullet$ female.

TABLE 2. Mean heart weight of cases over 30 years of age

\begin{tabular}{|c|c|c|}
\hline & $\begin{array}{c}\text { Number of cases } \\
\text { over age } 30\end{array}$ & $\begin{array}{l}\text { Mean heart weight (g) } \\
(95 \% \text { confidence interval) }\end{array}$ \\
\hline Cerebral hemorrhage & 40 & $422 \pm 28.5$ \\
\hline Subarachnoid hemorrhage & 17 & $359 \pm 34.8$ \\
\hline Cerebral infarction & 23 & $392 \pm 28.8$ \\
\hline Other diseases & 21 & $293 \pm 25.9$ \\
\hline
\end{tabular}




\section{Discussion}

It is today generally accepted that the frequency of cerebral hemorrhage against that of cerebral infarction is decreasing. According to Yates (1966), the ratio for cerebral hemorrhage in England and Wales has been falling in the last 30 years, while the rate of infarction has shown a remarkable rise after 1945, which means a reversal of the relative position of hemorrhage and ischemia as the major cause of fatal strokes. In Japan, Katsuki and Hirota (1966) also demonstrated a decline in the ratio of hemorrhage to infarction in their review based on the "Annual of Pathological Autopsy Cases in Japan" published by the Japanese Pathological Society. According to them, the frequency of cerebral thrombosis increased from $1.5 \%$ in 1958 to $2.5 \%$ in 1962 , whereas the frequency of cerebral hemorrhage remained $2.2 \%$. Consequently, the ratio of hemorrhage to infarction was estimated approximately at $1: 1$. In a later report, Hirota (1969) demonstrated on mortality statistics even a declining trend of cerebral hemorrhage, not only in the old age but also in the middle age. In contrast to these recent trends in the incidence of cerebrovascular strokes, our autopsy data suggest that primary intracranial hemorrhage still constitutes a major mortality factor in this district.

As regards the age distribution, Otsu (1969) reported that cases of cerebral hemorrhage were most frequent in 6 th and 7 th decades reviewing the 1601 autopsy statistics examined by his study group for cerebral apoplexy in Japan. Ooneda (1974) also analyzed 196 autopsy cases of cerebral hemorrhage at the Gunma University in a 21-year period from 1947 to 1967 and reported that the maximum incidence was found in the 6th decade. Compared with the result of these authors, the age distribution of the cerebral hemorrhage of our autopsy series was characterized by earlier onset of the disease.

With respect to the location of hematomas, the general trend of incidence was not essentially different from that hitherto reported, the predilection being the striate nuclei, where the arteries are predisposed to hypertensive injuries (Fukasawa 1969). Hemorrhages in the deep cerebral white matter, where hemodynamical predisposition of arterial lesions was unlikely, were found only in subjects of younger ages. This suggests some pathological changes other than angionecrosis (for example, cryptic arterio-venous malformations) in the younger patients, though they were not identified in the present study. Relatively high incidence of cerebellar hemorrhage in our series in comparison with other clinical statistical studies may be associated with the difficulty in diagnosing cerebellar hemorrhage, because its clinical symptoms are often similar to those of pontine hemorrhage.

Among the three types of strokes, the mean survival period was shortest for cerebral hemorrhage; but when compared with that reported by other authors, for instance by Stehbens (1972) who found that $87 \%$ out of 368 cases died within a week, the survival period of cerebral hemorrhage at our Institution is long. This is attributed to the introduction of NCU (neurological care unit) into the treatment of acute cases.

McCormick and Rosenfield (1973) recently proposed "reevaluation of the true 
relationship of systematic hypertension to strokes". According to these authors, a definite mechanism for hemorrhage could be identified in about two-thirds (95/ 144) of the patients with massive brain hemorrhage; they stressed the fact that only about one-fourth of their patients had hypertension as the only identified cause of the hemorrhage. They included, however, under the expression of massive brain hemorrhage, a variety of diseases such as leukemia and other bleeding disorders, ruptured saccular aneurysms, and brain neoplasms. In the present report, these were listed under the respective items; the percentage of brain hemorrhage caused by blood dyscrasias was actually lower than that of their data.

In this connection it is again emphasized that hypertension is the most significant etiological factor of spontaneous massive cerebral hemorrhage in this district. As in our autopsy cases the documentation of pre-ictal level of blood pressure was available in clinical histories, the rate of hypertensives was compared among the three cerebrovascular strokes and other diseases (Fig. 16). In about $74 \%$ of the cases of fatal cerebral hemorrhage there was definite hypertension, in $23 \%$ slight hypertension, and only $3 \%$ were normotensive. This relation was essentially the same for the group of cerebral infarction. In fatal SAH of aneurysmal origin, hypertension was regarded as an aggravating factor, and the difference in the rate of hypertensives between the group of SAH and that of the other diseases was also evident.

Hypertensive tendencies in the inhabitants of the northern part of Japan were already pointed out epidemiologically by Takahashi and his co-workers (1961); corroborative evidence of correlation between death from stroke and the frequency of hypertension was also presented in the present study both from the autopsy finding (heart weight) and from the clinical records. Our results support Kannel's

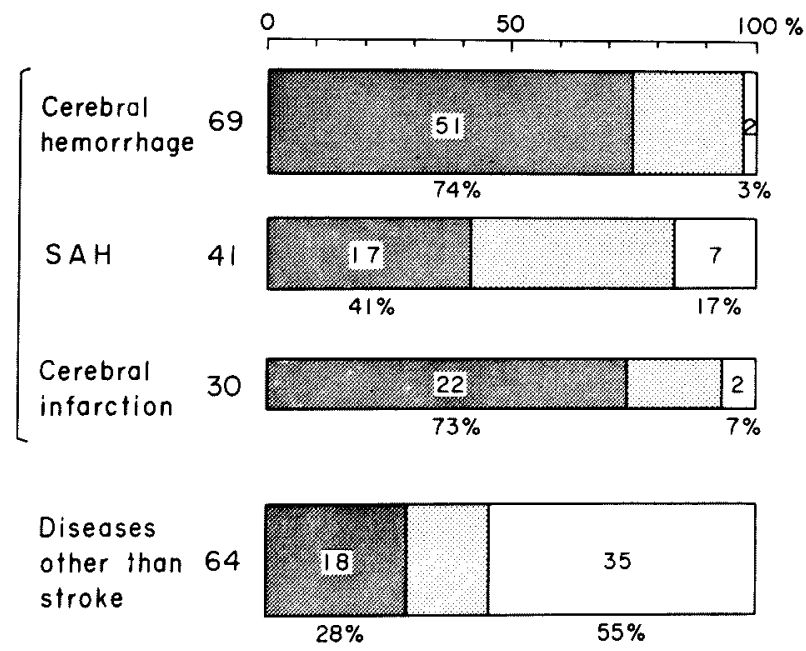

Fig. 16. Rates of hypertensives and non-hypertensives in the autopsy cases (204 consecutive cases). Definite hypertension ( $\mathrm{SBP} \geq 160 \mathrm{mmHg}$ or $\mathrm{DBP} \geq 95 \mathrm{mmHg}$ ); $\square$ slight or uncertain hypertension; $\square$ no evidence of hypertension. 
view (1966) that the risk of all types of stroke is distinctly elevated in hypertension.

\section{Acknowledgment}

I am grateful to Dr. T. Kutsuzawa, Director of the Hospital, and the other members of the hospital staff for providing clinical details. Technical assistance of Mr. M. Shirasawa, Mr. Y. Aoyama, Mr. S. Kato, Mr. Y. Tozawa and Mrs. Y. Minabe is appreciated.

\section{References}

1) Fukasawa, H. (1969) Hemodynamical studies of cerebral arteries by means of mathematical analysis of arterial casts. Tohoku J. exp. Med., 99, 255-268.

2) Hirano, A. (1961) Neuropathological study on ruptured intracranial aneurysms. Advances in Neurological Sciences (Jap.), 5, 480-499.

3) Hirota, Y. (1969) Epidemiological study on cerebrovascular diseases (CVD) and ischemic heart disease in Japan, based on mortality statistics, autopsy cases and population survey. Jap. Circulat. J., 33, 1467-1471.

4) Kannel, W.B. (1966) An epidemiologic study of cerebrovascular disease. In: Cerebral Vascular Diseases (Fifth Conference), edited by C.H. Millikan, R.G. Siekert \& J.P. Whisnant, Grune \& Stratton, New York, pp. 53-59.

5) Katsuki, S. \& Hirota, Y. (1966) Current concept of the frequency of cerebral hemorrhage and cerebral infarction in Japan. In: Cerebral Vascular Diseases (Fifth Conference), edited by C.H. Millikan, R.G. Siekert \& J.P. Whisnant, Grune \& Stratton, New York, pp. 99-105.

6) McCormick, W.F. \& Rosenfield, D.B. (1973) Massive brain hemorrhage: a review of 144 cases and an examination of their causes. Stroke, 4, 946-954.

7) Ooneda, G. (1974) Noshukketsu no Byori (Jap.), Bunkodo, Tokyo, p. 11., pp. 20-91.

8) Ooneda, G., Yoshida, Y., Suzuki, K. \& Sekiguchi, T. (1973) Morphogenesis of plasmatic arterionecrosis as the cause of hypertensive intracerebral hemorrhage. Virchows Arch. Abt. A, Path. Anat., 361, 31-38.

9) Otsu, S. (1969) The incidence of cardio- and cerebrovascular disease in autopsy cases. Jap. Circulat. J., 33, 1459-1465.

10) Stehbens, W.E. (1972) Pathology of the Cerebral Blood Vessels, C.V. Mosby Co., Saint Louis, pp. 284-350.

11) Takahashi, E., Kato, K., Kawakami, Y., Ishiguro, K., Kaneta, S., Kobayashi, S., Ohba, E., Yano, S., Ito, Y., Shiraishi, M., Murakami, N., Sugawara, T., Meguro, Y. \& Suzuki, Y. (1961) Epidemiological studies on hypertension and cerebral haemorrhage in North-east Japan. Tohoku J. exp. Med., 74, 188-210.

12) Yates, P.O. (1966) The changing pattern of cerebrovascular disease in the United Kingdom. In: Cerebral Vascular Diseases (Fifth Conference), edited by C.H. Millikan, R.G. Siekert \& J.P. Whisnant, Grune \& Stratton, New York, pp. 67-73. 